Financial Markets for Unknown Risks

by

Graciela Chichilnisky, Columbia University

Geoffrey Heal, Columbia Business School

January 1992, Revised August 1992

Discussion Paper Series No. 642

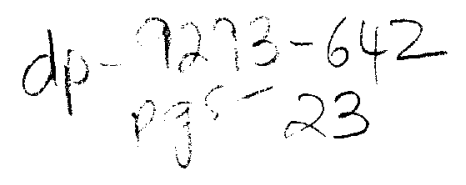




\title{
Financial Markets for Unknown Risks
}

\author{
Graciela Chichilnisky* \\ Columbia University
}

\author{
Geoffrey Heal \\ Columbia Business School
}

January 1992 revised August 1992

\begin{abstract}
An economy faces an unknown individual risk, such as the health effects of a recently discovered environmental hazard. Opinions may be widely different about the distribution of risks across the population. We study financial markets that suffice to reach efficient allocations in this situation. The problem is formalized in a general equilibrium economy with incomplete markets for individual and collective uncertainty. We show that ignorance of the probabilities describing individual risk leads to collective risk. Introducing an array of mutual insurance policies and of Arrow securities is shown to lead to Arrow Debreu competitive allocations. By combining insurance contracts for individual risks and securities markets for collective risks, the proposed institutional framework economizes significantly on the number of markets required for efficiency. The computational complexity of a market equilibrium is reduced from an NP - complete (i.e. intractable) problem to one which depends polynomially on the number of households.
\end{abstract}

JEL Classification Number: G14, D50, G33,

Key words: collective risk, individual risk, insurance, financial markets, incomplete markets, unknown risks, intractable, NP complete.

\section{Contents}

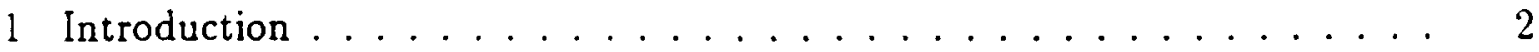

2 Markets for unknown individual risks. . . . . . . . . . . . . . . 4

3 An Economy with Individual and Collective Risks . . . . . . . . . . . 6

4 The Behavior of Households . . . . . . . . . . . . . . . . 8

j Efficient allocations ................... . . . 10

6 Equilibrium \& incomplete markets for unknown risks . . . . . . . . . 13

T Efficient allocations, mutual insurance \& securities . . . . . . . . . . . 14

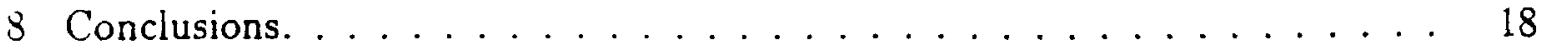

- We are grateful to David Cass for valuable comments on an earlier version of this paper. 


\section{Introduction}

Consider an economy in which each household faces an individual risk with an unknown probability. Such risks derive, for example, from the effects on health of ozone depletion, of acid rain, or of air pollution. The risks might be not just unknown but also unknowable, in the sense that we cannot reasonably imagine repetition of the harmful events a sufficient number of times to permit estimation of probabilities. Clearly this is the case with ozone depletion or global warming, which are events that will only happen once if at all. In such cases, opinions may differ widely about the magnitudes of the risks to which individuals are exposed. What market structure would suffice to assure efficient allocations in this situation?

This question is formalized in a general equilibrium model. Each household faces the risk of being in one of $\mathrm{S}$ states (e.g. healthy or sick). It has different endowments in each state. The risks are unknown, in the sense that no-one knows the true distribution of affected individuals in the population. A probability distribution is assigned to each possible frequency distribution over the population. This distribution over distributions may be different from individual to individual

We have therefore two levels of uncertainty. The first level of uncertainty is collective: what is the distribution of affected individuals in the economy. The second level of uncertainty is individual: it is uncertainty about whether a given household is sick or not. Consider as illustrations the impact of depletion of the ozone layer on skin cancer, or of acid rain and air pollution on respiratory diseases. There are widely differing opinions about these impacts, on which there is inadequate information. In some cases, the inherent uniqueness of the events concerned makes it impossible to develop a statistical basis for risk assessment. Then the two types or levels of uncertainty are, firstly, uncertainty about the true impact of ozone depletion, acid rain or air pollution on individual disease (and therefore about the incidence in the population as a whole), and, secondly, uncertainty about whether any given person will develop a disease. It is this ignorance of the basic scientific processes (e.g., the relation between ozone depletion and skin cancer) that causes the collective risk, i.e., uncertainty about the distribution of affected individuals in the population.

We know of course that a complete set of state-contingent commodity markets would lead to a Pareto efficient allocation. However, this approach may be impracticable as the number of markets needed with individual risks is shown below to rise exponentially with the number of agents in the economy. This is because contingent commodity markets are a very cumbersome way of dealing with individual risks: they require decisions over an exhaustive enumeration of all possible combinations of individuals and states over the population. We propose an alternative institutional framework which uses only two types of instruments, and which economizes significantly on the number of markets needed. One is a mutual insurance contract to deal with the individual risk contingent on each possible distribution of harmful effects in the population. Secondly, we need Arrow securities to deal with the collective risk 
induced by ignorance, i.e. the uncertainty about the overall distribution of adverse effects in the economy. One Arrow security is needed for each possible distribution in the population, because to attain Pareto efficiency each possible distribution must be treated as a separate (collective) state. Depending on the state of knowledge, these may be limited to a relatively small number of distributions, each described, for example, by an approximate percentage of affected individuals.

In informal terms, our approach can be described as follows. We group together all states of the economy which are statistically identical in the sense that the distribution of agents by individual state (sick, healthy, etc.) is the same in each. We call the resulting statistical descriptions of the economy statistical states. These describe precisely the aggregate statistical characteristics of the economy, but leave open the status of any given individual. We assume that every distribution of risk across the population having the same statistical description is equally probable. We then show in Theorem 1 that Arrow-Debreu equilibrium allocations can be supported as follows. Households buy Arrow securities each of which pays in a specific statistical state. For each statistical state, the quantity of Arrow securities purchased pays an amount equal to the expected difference between the value of consumption at the equilibrium allocation to be supported, and the value of endowments at that equilibrium. The expectation is conditional on being in the specified statistical state. So the Arrow securities purchased assure that on average over all possible individual states in each statistical state, households' budgets balance. Of course, this still leaves the possibility that in any particular individual state there is a shortfall or excess of income over expenditure. The role of mutual insurance contracts is then to guarantee to make good the shortfalls, in exchange for the excesses as premia. The paper proves that such an intuitively clear procedure can be made to work consistently, at Arrow-Debreu allocations. We show that markets for Arrow securities clear, and that insurance contracts of this type are actuarially fair. Theorem 2 then shows that the problem of reaching an efficient allocation of resources with complete ArrowDebreu contingent commodity markets is intractable with respect to the number of agents in the economy, whereas with the proposed alternative structure this problem is tractable. A novel theoretical insight into the structure of Arrow-Debreu equilibria is provided by Propositions 1 and 2. These show that in large economies there are equilibrium prices which depend only on statistical properties of the economy. These propositions establish that under certain conditions, equilibrium prices are the same for all statistically identical realizations of the states of individuals in the economy. Propositions 1 and 2 are used in establishing Theorem 1.

There are two features of the results which are of general interest. One is the development of a framework for achieving efficient allocations in the face of individual risks whose probabilities are unknown. Given rapid changes in technology with potentially far-reaching environmental impacts and health effects, the problem of providing insurance against unknown risks is particularly important (Heal [12]). It is a matter of very active concern in the insurance industry. 
The second interesting feature is the way a combination of securities markets and insurance markets can be used to simplify the institutional structure needed for dealing with unknown individual risks. The point here is that unknown individual risks can be resolved into a collective and an individual component. The collective component refers to the distribution of certain conditions in the population as a whole, and the individual component, to the probability of a particular person being affected. Securities markets are used to insure against the collective component, and insurance contracts are used for the individual components. This is illustrated in Figure 1. Typically securities markets have not been seen as appropriate for handling individual risks, and indeed the use of securities markets and insurance markets together has not previously been studied (except for Cass, Chichilnisky and Wu [3]). Given the current interest in the insurance industry about its relationship with the securities industry, a model of value-added interaction between the two is very timely.

The present paper draws on recent findings of Chichilnisky and Wu [4] and Cass, Chichilnisky and $\mathrm{Wu}[3]$, both of which study resource allocation with individual risks. Both of these papers develop further Malinvaud's [13] [14] original formulation of general equilibrium with individual risks, and Arrow's [1] formulation of the role of securities in the optimal allocation of risk-bearing. Our results are valid for large but finite economies with several types of agents who face unknown risks and who have diverse opinions about these risks: in contrast, Malinvaud's results are asymptotic, valid for a limiting economy with an infinite population, and deal with identical individuals with a known and identical distribution for each. Our results use the formulation of incomplete asset markets for individual risks used to study default in Chichilnisky and Wu [4], Section j.c. The risks considered here are unknown and possibly unknowable, and each individual has potentially a different opinion about these risks, while Chichilnisky and Wu [4] and Cass, Chichilnisky and Wu [3] assume that all risk is known. [3] also assumes a neat separation between individual and collective risks, so that collective risks are always resolved before individual risks. There is no interaction between the two types of risk. Our formulation here does not assume such separation: it does however reduce collective risk to distributions over proportions of people in each state, i.e., distributions over degenerate distributions. In addition, we prove here that Arrow-Debreu competitive equilibrium allocations can always be obtained by introducing an array of mutual insurance contracts and Arrow securities in an incomplete asset market, while Cass, Chichilnisky and Wu [3] focus instead on the assets required to decentralize the Pareto efficient allocations of an economy akin to Malinvaud's [14] market organization type B.

\section{Markets for unknown individual risks.}

Uncertainty is represented by random variables which describe nature's. moves. Each realization of a random variable is a state. Collective states are realizations of random variables that affect simultaneously all individuals in the economy, such as the 
Two alternative proportions A \& B of affected individuals in the population, i.e., two collective states A \& B.

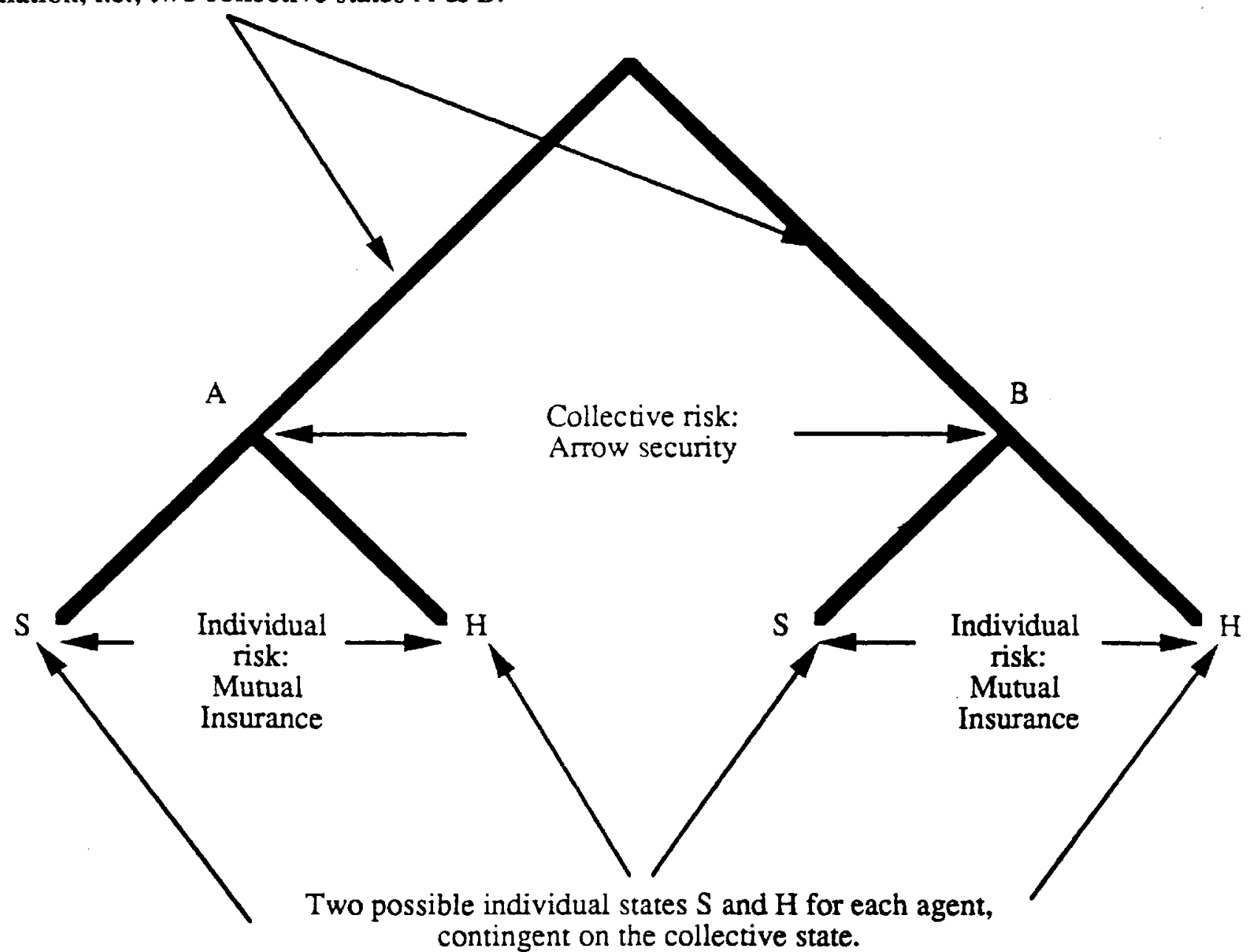

Figure 1: the realtionship between individual and collective states, and the use of securities and mutual insurance contracts. 
weather. Individual states are realizations of a random variable that affects one individual in the economy, such as their health. We assume here that households' endowments depend on their individual states (healthy or sick) only, implying that individuals face individual risk.

The markets required to obtain Pareto efficient allocations are very different depending on whether there is collective or individual risk. Insurance is the asset of choice for allocation of risk-bearing in a large economy with individual risk (see Arrow and Lind [2], Malinvaud [13] [14], Chichilnisky and Wu [4] and Cass, Chichilnisky and Wu [3]) while contingent contracts (Debreu [7]) or securities (Arrow [1]) are the assets used for the same purpose in economies with collective risk. This paper formalizes the relationship between these two different types of risks, and the assets for dealing with unknown individual risks.

Standard definitions of individual risk [13] require that as populations increase, the proportion of people in each state (e.g. sick or healthy) converges to a fixed number about which there is no uncertainty. This is the law of large numbers. Working in the limit and assuming known risks ensures that the statistical state is known in his model, and this is a crucial element in his analysis.

Instead our economy is large but finite, and here no-one knows for sure the true distribution of risks in the population, i.e., the true statistical state. Furthermore different individuals may have different opinions about the matter. In this situation, mutual insurance contracts could be sold at premia contingent on the realization of the statistical state. However, even if all households purchase such insurance against their unfavorable states, they still face a remaining uncertainty about the true probability distribution that governs their contracts, and therefore about the premium they must pay and the payments which they will receive. They could use such contracts to insure their individual risks conditional on the statistical state of the economy, and so still face uncertainty about the statistical state. For each household, this latter uncertainty is uncertainty about economic variables such as prices, a subject central to the analysis of Chichilnisky and Wu [4], Chichilnisky, Dutta and Heal [5] and Chichilnisky, Heal, Streufert and Swinkels [6]. The uncertainty derived from ignorance is collective rather than individual: it refers to distributions of people across states in the whole economy, and it affects everyone. It is therefore amenable to Arrow's [1] or Debreu's [7] treatment of markets for efficient allocation of risk-bearing.

In practical terms, this means that when individual risks are unknown, the economy has collective as well as individual risks. We construct a market organization which economizes on the number of assets required to hedge against all risk, consisting of mutual insurance markets to deal with the individual risks, and of Arrow securities to deal with the collective risk induced by ignorance. We show that this leads to Arrow-Debreu equilibrium allocations, and thus to Pareto efficiency.

In principle, efficiency requires as many contingent markets as the number of all collective states $\sigma$, namely the number of lists of all possible individuals in all possible states. Each such list is called a collective state. With $\mathrm{H}$ individuals in total, made 
up of $H_{i}$ of each of $H$ types, and $\mathrm{S}$ individual states this number is $S^{H}$, a number which increases exponentially with the number of people in the economy. Having $N S^{H}$ contingent markets, one for each good and for each state $\sigma$, leads to an ArrowDebreu model when households act competitively, and to Pareto efficient allocations when markets clear. However, we shall show that under our assumptions, there is a large amount of duplication of contingent markets or securities. This duplication can be avoided by considering, rather than the $S^{H}$ collective states $\sigma \in \Omega$, statistical states $\mathrm{r}$ which represent the proportions of people of different types who are in different states. This is an anonymous representation of collective states, which provides the same economic information without revealing the actual identities of the individuals. There are $A=\prod_{i}\left(\begin{array}{c}H_{0}+S+1 \\ S-1\end{array}\right)$ statistical states, and $S$ individual states. We show that under the assumptions made on probabilities and utilities, an array of I mutual insurance contracts, one for each type $i$, and one Arrow security for each statistical state $r \in R$, will suffice to reach efficient allocations. This economizes substantially on markets: it decreases the number of markets needed from $N S^{H}$ to $N+I A+A$. This is the subject of Theorem 1 .

For example, with $\mathrm{N}$ goods, 100 individuals of two types, half of each type, and 2 individual states, the number of Arrow-Debreu markets needed to achieve Pareto efficiency is $\mathrm{N} 2^{H}=\mathrm{N} 2^{100}$, or $\mathrm{N}$ spot markets and $2^{100}$ Arrow securities markets with perfect price foresight. Theorems 1 and 2 show that in this example, it suffices to have $\mathrm{N}$ spot markets, plus 2 mutual insurance contracts, and 2809 Arrow securities. Theorem 2 quantifies this reduction, showing that the computational complexity of checking for an equilibrium rises exponentially with the number of agents in the Arrow-Debreu framework, i.e. it is an intractable problem, while in our framework the same complexity is polynomial so that the problem is tractable. This saving in the number of markets can be achieved because at every collective state $\sigma$ leading to a given statistical state $r$, i.e. $r(\sigma)=r$, the total endowments of the economy are the same, and there is the same number of individuals of each type in each state, each with the same endowments and utilities. To prove that efficient allocations can be achieved with this reduced number of markets, we use crucially the assumption that endowments depend solely on individual states, and that utilities depend solely on consumption at a combination of statistical states and individual states. We also use the fact that every household $h$ of type $i$ has the same probability distribution $\Pi^{i}$ over collective states, and that all these probability distributions satisfy an anonymity requirement, defined in Section 3.

\section{An Economy with Individual and Collective Risks}

The distinction between collective and individual risk is by no means always as clear cut as may appear from the names. In extreme cases it is simple. One extreme case is the case of individual risks that are identically and independently distributed: here collective risk is determined from individual risk and individual risk is the basic risk. 
Another extreme case is that of fully correlated risks, as normally considered in the Arrow-Debreu model. Purely individual or purely collective risks are extreme cases which help to organize our thinking. just as are pure private goods and pure public goods in another field. In general we can think of IID individual risks (as considered in Malinvaud [13]) as one polar case in a continuum that ranges to the other extreme of fully correlated risks. Cass. Chichilnisky and $\mathrm{Wu}[3]$ consider a different case, as explained above. Uncertainty about the nature of individual risk, which may take several values, is one cause of collective risk. It is this case that we model here.

Consider an exchange economy with.$V$ consumption goods. There are $H$ households, divided into types indexed by $i=1, \ldots, I$ and $H_{i}$ households of type $\mathrm{i}$, so that $H=\sum_{i} H_{i}$. Each household faces the same set of $S$ individual states, indexed by $s=1, \ldots, S$. All households of type $i$ have the same utilities and endowments: the latter depends solely on the household's individual state $s$, representing individual risk. Let $z_{\text {jho }}$ denote the quantity of good $j$ consumed by household $h$ in collective state $\sigma$ : $z_{h \sigma}$ is an $N$ dimensional vector $z_{j h \sigma}, j=1, \ldots, N$ and $z_{h}$ an $N S^{H}$ dimensional vector $z_{h \sigma}, \sigma \in \Omega$. All consumption vectors are assumed to be non - negative. We now define the set of collective states, $\Omega=\{\sigma: \sigma=$ a function from $\{1, \ldots, H\}$ into $\{1, \ldots, S\}\} . \Omega$ consists of all possible lists of the $\mathrm{H}$ individuals' states; it has $S^{H}$ elements. Let $s(h, \sigma)$ be the state of individual $h$ in the collective state $\sigma$. Let $r_{i s}(\sigma)$ be the proportion of all households $h$ of type $\mathrm{i}$ for whom $s(h, \sigma)=s$. Then $\sum_{i} \sum_{s} r_{i s}(\sigma)=I$. Let $r_{i}(\sigma)=r_{i 1}(\sigma), \ldots, r_{i S}(\sigma)$ be the distribution of households of type $i$ among individual states within the collective state $\sigma$, and let $r_{s}$ be the proportion of all individuals in state $s$. Similarly let $r(\sigma)$ be the proportion of households of each type in each individual state, $r(\sigma)=r_{1}(\sigma), \ldots, r_{I}(\sigma)$. Let $R$ be the set of vectors $r(\sigma)$ when $\sigma$ runs over $\Omega ; r=r(\sigma) \in R$ is a statistical state. It is called a statistical state because it is defined only by the total number of individuals in each state for each type, and does not contain any information about the identities of the individuals themselves. $R$ is contained in $S^{I}$, the product of $I S$-dimensional simplices, and has $\prod_{i}\left(\begin{array}{c}H_{i}+S-1 \\ S-1\end{array}\right)$ elements.

We assume that collective uncertainty is about statistics. This means that each possible distribution of risks in the population as a whole, gives measure one to the set of all states leading to a particular statistical state. When interpreted as a distribution over statistical states, such a distribution gives measure one to a particular statistical state, i.e., to a particular set of proportions $r(\sigma)$ of affected individuals in the population. It is a point distribution over the set $R$ of statistical states, identifying a particular statistical state. This has the following important implication: an individuals' probability distribution over possible distributions of risks in the population as a whole, is in fact a distribution over statistical states. This is because each possible distribution of risks over the population is a statistical state. Figures 2 and 3 illustrate the relationship between collective and statistical states, and the concentration of the supports on single statistical states.

This is an assumption of convenience only, avoiding the mechanical complexity 


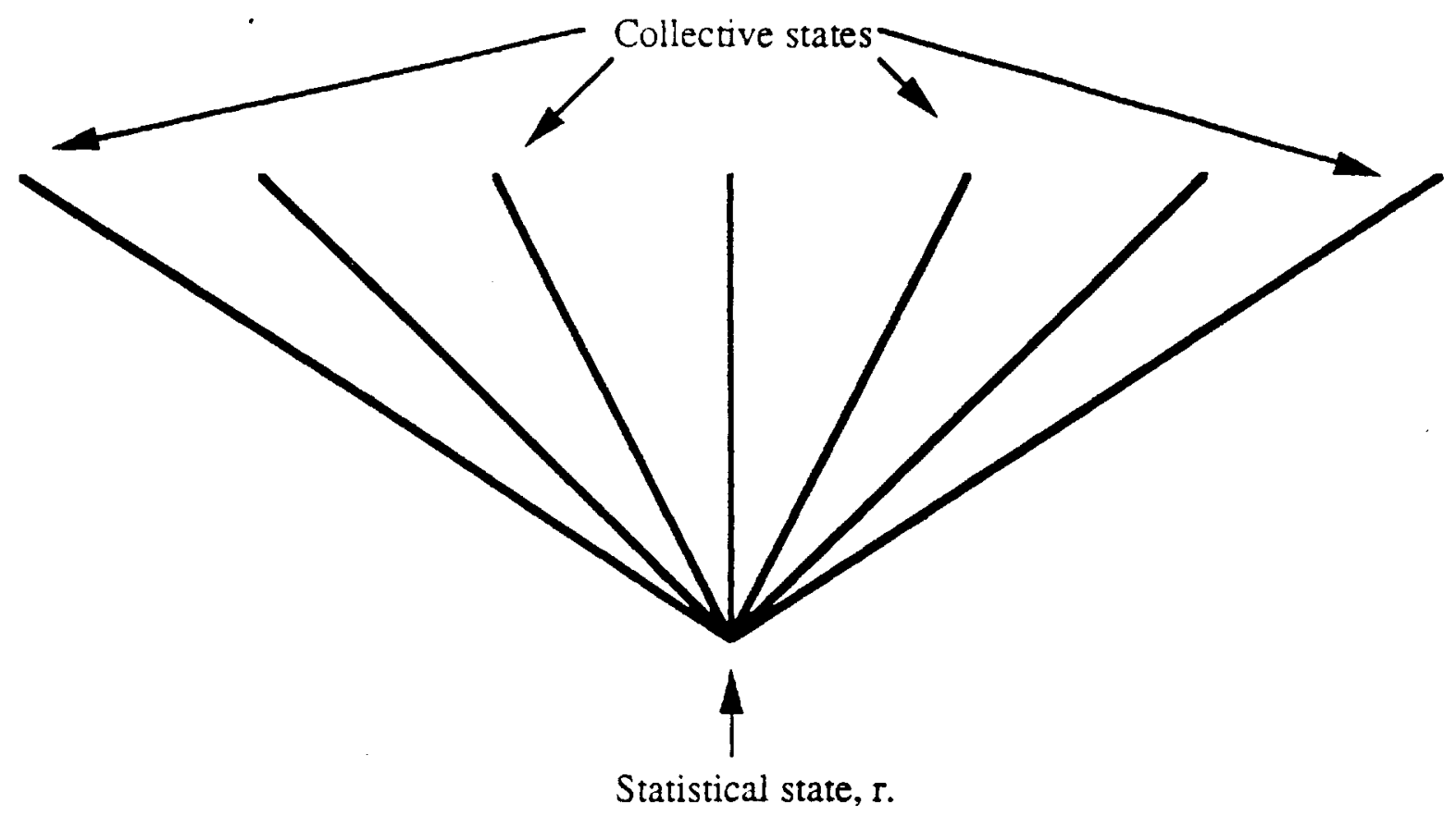

Figure 2: many collective states give rise to the same distribution of affected individuals, i.e., to the same statistical state $r$.

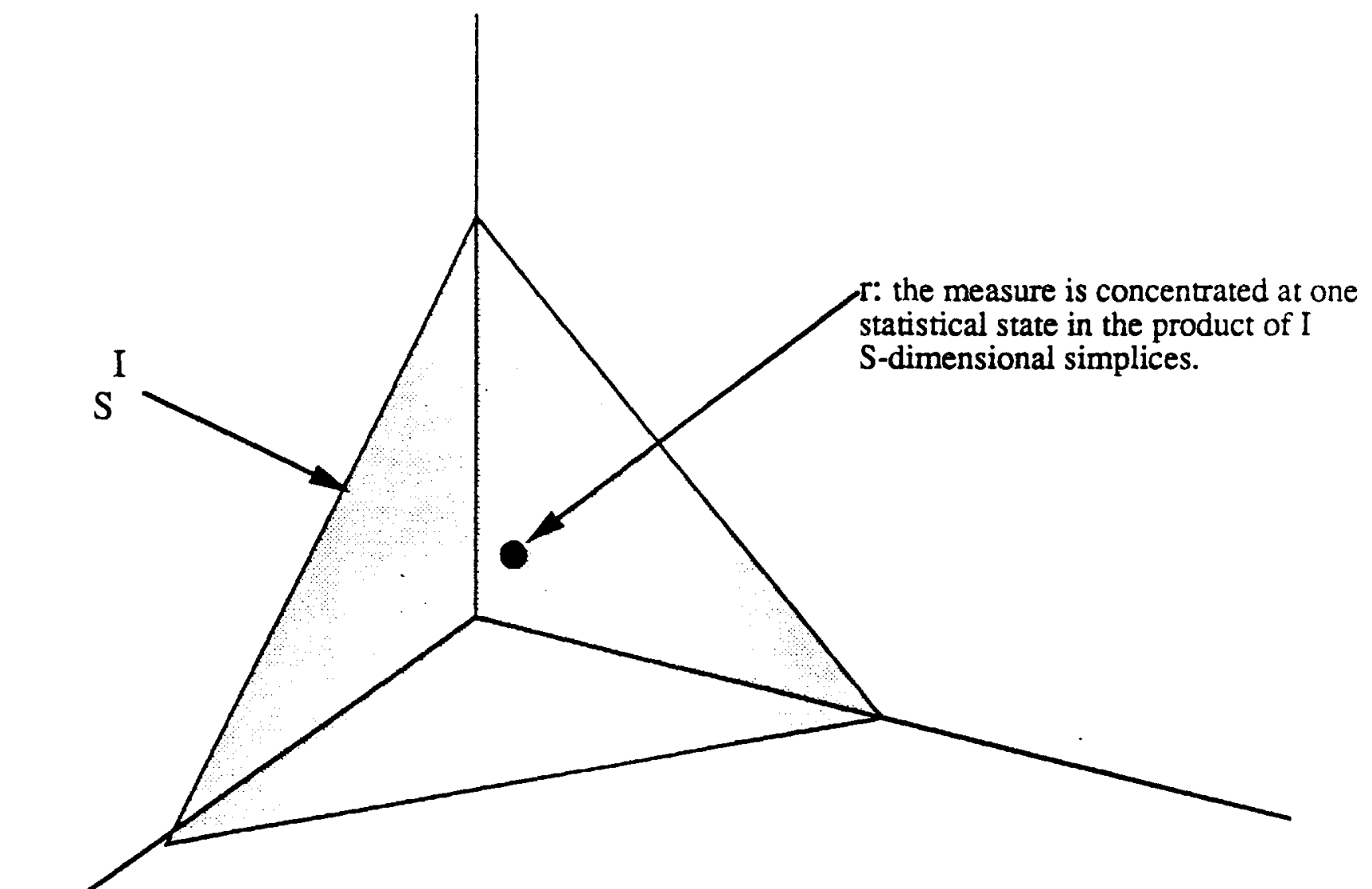

Figure 3: possible distributions over statistical states are point distributions. 
of dealing with infinite economies. An alternative way of avoiding such complexities is given in Cass, Chichilnisky and $\mathrm{Wu}$ [3], who consider the case of known risks. In their analysis there is no ignorance of the underlying process. Their collective risk is given by a distribution not necessarily supported on one proportion. They assume the process generating the individual states to be such that $\Pi_{\mathrm{s} / \mathrm{r}}^{i}=r_{i s}$, and justify this by reference to a model where "nature (or in the older tradition God) draws randomly twice: First to determine how many balls to take out of the urn, and then second, to determine which specific balls are actually withdrawn." This approach requires a two-stage realization of the underlying random process, with stage one revealing the statistical state and then stage two revealing each agent's state. Our approach here allows more general models of uncertainty in which risks are unknown.

We define next probability distributions $\Pi^{i}$ on the set of all collective states, one for each type of household, $i=1, \ldots, I: \Pi^{i}$ is type $i$ 's probability distribution over the set of collective states $\Omega$, and $\Pi_{\sigma}^{i}$ denotes the probability of state $\sigma$. Types are therefore characterized by both probability distributions over collective states and by the endowments that they have in different individual states. The statements made about $\Pi^{i}$ from now on, are valid for all $i$.

The following anonymity assumption is required:

$$
r(\sigma)=r\left(\sigma^{\prime}\right) \rightarrow \Pi_{\sigma}^{i}=\Pi_{\sigma^{\prime}}^{i}
$$

This means that two distributions which have the same statistical characteristics are equally likely. Then $\Pi_{\sigma}^{i}$ defines a probability distribution $\Pi_{r}^{i}$ on the space of statistical states $R ; r_{i s}$, as above, gives the proportions in $r$ of households $h$ of type $i$ which are in individual state $s$. $\Pi_{r}^{i}$ can be interpreted, as remarked above, as $i$ 's distribution over possible distributions of affects in the population as a whole. Malinvaud [14] p. 38T, para. I notes that the probability that a statistical state $r$ obtains and that simultaneously, for a given household h of type $i$, a particular state $s$ also obtains is

$$
\Pi_{s r}^{i}=\Pi_{r}^{i} r_{i s} \text { with } \sum_{s} \Pi_{s r}^{i}=\Pi_{r}^{i}
$$

The probability $\Pi_{j}^{i}$ that, for a given $h$ of type $i$, a particular individual state $s$ obtains is therefore given by

$$
\Pi_{s}^{i}=\sum_{r \in R} \Pi_{r}^{i} \cdot r_{i s}
$$

\section{The Behavior of Households}

Let $e_{s \sigma}^{i}$ be the endowment of a household of type i when the collective state is $\sigma$ and the individual state is $\mathrm{s}$. Since all households $\mathrm{h}$ of type $\mathrm{i}$ have the same endowment in the individual state $\mathrm{s}$, we can write their endowment $e_{s \sigma}^{i}=e_{3}^{i}$ in any collective state $\sigma$ with $s(h, \sigma)=s$. Let $z_{h}^{i}$ be the $N S^{H}$ dimensional vector indicating the 
consumption of all goods across collective states of household h of type i. Similarly, let $z_{h \sigma}^{i}$ indicate the components of this vector within a given collective state $\sigma \in \Omega$. Type $i$ individuals are assumed to have the same probabilities $\Pi_{\sigma}^{i}$ for each $\sigma$, and the same von Neumann-Morgenstern utility:

$$
W^{i}\left(z_{h}^{i}\right)=\sum_{\sigma} \Pi_{\sigma}^{i} U_{s(h, \sigma)}^{i}\left(z_{h \sigma}^{i}\right)
$$

where as defined above $s(h, \sigma)$ is the individual state $s$ of household $h$ within the collective state $\sigma$. This definition of $W^{i}\left(z_{h}^{i}\right)$ indicates that household $\mathrm{h}$ of type $\mathrm{i}$ has preferences on consumption which may be represented by a "state separated" utility function $W^{i}$ defined from $S$ elementary utility functions $U_{s}^{i}$. Note that in state $\sigma$, $U_{s}^{i}=U_{s(h, \sigma)}^{i}$. The functions $U_{s}^{i}$ are assumed to be $C^{2}$, strictly increasing, strictly quasiconcave, and the closure of the indifference surfaces $\left\{U_{s}^{i}\right\}^{-1}(x) \subset$ int $\left(R^{N+}\right)$ for all $x \in R^{+}$. The probabilities $\Pi_{\sigma}^{i}$ are in principle different over household types $i$.

We shall assume like Malinvaud [13] that preferences are separable over statistical states. This means that the utility of household $h$ depends on $\sigma$ only through the statistical state $r(\sigma)$. If we assume further that in state $\sigma$ household $h$ takes into account only what happens to it, i.e. $s(h, \sigma)$, and what frequency distribution $r(\sigma)$ happens to appear, but nothing else, then the consumption plan can be expressed as $z_{\sigma}^{i}=y_{s(h, \sigma)}^{i}(r(\sigma))$, which in the following is also denoted $y_{s r}^{i}$. The summation with respect to collective states $\sigma$ can now be made first within each statistical state. To a particular statistical state $r$ and individual state $s$ for which there is a non - zero incidence of individuals in individual state $s$, i.e. $r_{s} \neq 0$, there correspond a number of collective states $\sigma$ leading to $r(\sigma)=r$ and $s(h, \sigma)=s$, hence to the same $U_{s(h, \sigma)}^{i}\left(z_{h \sigma}^{i}\right)=U_{s}^{i}\left(y_{s r}^{i}\right)$. All individuals of type $i$ have the same probability distribution over statistical states $\Pi_{r}^{i}$ and thus the same joint and conditional probability distributions $\Pi_{s r}^{i}$ and $\Pi_{s / r}^{i}$ for all $s, r$ (though individuals of different types may have different probabilities). Hence for the household h of type $i, W^{i}$ may also be written as

$$
\begin{gathered}
W^{i}\left(z_{\sigma}^{i}\right)=W^{i}\left(y_{s r}^{i}\right)=\sum_{r \in R_{H}} \Pi_{r}^{i} \sum_{s=1}^{S} r_{i s} U_{s}^{i}\left(y_{s r}^{i}\right) \\
=\sum_{r, s} \Pi_{r}^{i} \Pi_{s / r}^{i} U^{i}\left(y_{s r}^{i}\right)=\sum_{r, s} \Pi_{s r}^{i} U^{i}\left(y_{s r}^{i}\right)
\end{gathered}
$$

which expresses the utility of a household in terms of its consumption at individual state $s$ within a statistical state $r$. This expression is important in the following results, because it allows us to represent the utility of consumption across collective states $\sigma$ as a function of statistical states $r$ and individual states $s$ only. This underlies the proof that it suffices to cover individuals against their individual risks $s$, and statistical risks $r$. 


\section{$5 \quad$ Efficient allocations}

Let $p^{*}$ be a competitive equilibrium price vector of the Arrow-Debreu economy $\mathrm{E}$ with markets contingent on all collective states (defined below) and let $z^{*}$ be the associated allocation. We will as usual say that $z^{*}$ is Pareto efficient if it is impossible to find an alternative feasible allocation which is preferred by at least one agent and to which no agent prefers $z^{*}$. Let $p_{\sigma}^{*}$ and $z_{\sigma}^{*}$ be the components of $p^{*}$ and $z^{*}$ respectively which refer to goods contingent on state $\sigma$. Proposition 1 considers the case when households agree on the probability distribution over collective states, denoted $\Pi$. It follows that they agree on the distribution over statistical states. It shows that in this case, the competitive equilibrium prices $p^{*}$ and allocations $z^{*}$ are the same across all collective states $\sigma$ leading to the same statistical state $r{ }^{1}$ Define now an Arrow-Debreu economy denoted $E$, where markets exists contingent on an exhaustive description of all states in the economy, i.e. for all collective states $\sigma \in \Omega$. We therefore have $N S^{H}$ contingent markets. An Arrow-Debreu equilibrium is a price vector $p^{*}=\left(p_{\sigma}\right), p_{\sigma} \in R^{N+} \sigma \in \Omega$, and an allocation $z^{*}$ consisting of vectors $z_{h}^{*}=\left(z_{h \sigma}^{*}\right), z_{h \sigma}^{*} \in R^{N+}, \sigma \in \Omega, h=1, \ldots H$ such that for all $h, z_{h}^{*}$ maximizes

$$
W^{h}\left(z_{h}\right)=\sum_{\sigma} \Pi_{\sigma}^{h} U^{h}\left(z_{h \sigma}^{*}\right)
$$

subject to a budget constraint

$$
p\left(z_{h}^{*}-e_{h}\right)=0
$$

and all markets clear:

$$
\sum_{h}\left(z_{h}^{*}-e_{h}\right)=0
$$

Proposition 1 Consider an Arrow-Debreu equilibrium of the economy $E, p^{*}=\left(p_{\sigma}^{*}\right)$, $z^{*}=\left(z_{\sigma}^{*}\right), \sigma \in \Omega$. Assume that all households have the same probability distribution over statistical states, i.e., for all $i . j, \Pi_{\sigma}^{\prime}=\Pi_{\sigma}^{j}=\Pi_{\sigma}$. Then for every state $\sigma$ leading to a given statistical state $r$, i.e., $r(\sigma)=r$, equilibrium prices and consumption allocations are the same, i.e., there exists a price vector $p_{r}^{*}$ and an allocation $z_{r}^{*}$ such that $p_{\sigma}^{*}=p_{r}^{*}$ and $z_{\sigma}^{*}=z_{r}^{*}$, where $p_{r}^{*} \in R^{N+}$ and $z_{r}^{*} \in R^{N I}$ depend solely on $r$. Furthermore, any two households $h$ and $k$ of the same type $i$ have the same equilibrium consumption vectors, i.e., $z_{h r}^{*}=z_{k r}^{*}=z_{r}^{i *}$.

Proof. Consider $\sigma_{1}$ and $\sigma_{2}$ with $r\left(\sigma_{1}\right)=r\left(\sigma_{2}\right)=r$. Note that the total endowments of the economy are the same in $\sigma_{1}$ and $\sigma_{2}$, both equal to $s_{r}=\sum_{i} H_{i} r_{i s} e_{s}^{i}$. Also, by the anonymity assumption. $\Pi_{\sigma_{1}}=\Pi_{\sigma_{2}}=\Pi_{r}$, where $\Pi_{r}$ is the probability of any social state in the statistical state $r$. We now show that for every household $h$, $z_{h \sigma_{1}}^{*}=z_{h \sigma_{2}}^{*}$, due to the Pareto efficiency of Arrow-Debreu equilibria. Assume not.

${ }^{1}$ Related propositions were established by Malinvaud in an economy where all agents are identical, and risks are known. 
Let $\Omega_{r}=\{\sigma: r(\sigma)=\sigma\}$. Let $z^{*}=\left(z_{h \sigma}^{*}\right)$, and assume there are $\sigma_{1}$ and $\sigma_{2} \in \Omega_{r}$ such that $z_{h \sigma_{1}}^{*} \neq z_{h \sigma_{2}}^{*}$ for some $h$. Define $E z_{h r}=\sum_{\sigma \in \Omega_{r}} z_{h \sigma}^{*} \frac{\Pi_{\sigma}}{\Pi_{r}}$. This is the expected value of $\left(z_{h \sigma}^{*}\right)$ given that the economy is in the statistical state r. Now

$$
\sum_{h} E z_{h r}=\sum_{h} \sum_{\sigma \in \Omega_{r}} z_{h \sigma}^{*} \frac{\Pi_{\sigma}}{\Pi_{r}}=\sum_{h} z_{h o}^{*}
$$

so that $E z_{h \sigma}$ is a feasible consumption vector for each $h$ in the aggregate collective state $r$. Next we show that by strict concavity, moving for each $h$ and each $\sigma$ from $z_{h \sigma}^{*}$ (which depends on $\sigma$ ) to $E z_{h r}$ (which is the same for all $\sigma \in \Omega$ ), is a strict Pareto improvement. This is because

$$
W^{h}\left(z_{h \sigma}^{*}\right)=\sum_{\sigma} \Pi_{\sigma} U^{h}\left(z_{h \sigma}^{*}\right)=\sum_{r} \Pi_{r} \sum_{\sigma \in \Omega} \frac{\Pi_{\sigma}}{\Pi_{r}} U^{h}\left(z_{h \sigma}^{*}\right)
$$

Hence by strict concavity of preferences,

$$
\sum_{r} \Pi_{r} \sum_{\sigma \in \Omega_{r}} \frac{\Pi_{\sigma}}{\Pi_{r}} U^{h}\left(z_{h \sigma}^{*}\right)<\sum_{r} \Pi_{r} \sum_{\sigma \in \Omega_{r}} U^{h}\left(\sum_{\sigma \in \Omega} z_{h \sigma}^{*} \frac{\Pi_{\sigma}}{\Pi_{r}}\right)=\sum_{r} \Pi_{r} \sum_{\sigma \in \Omega} U^{h}\left(E z_{h \sigma}\right)
$$

Since $E z_{h S}$ is Pareto superior to $z^{*}$ with $z_{h \sigma_{1}}^{*} \neq z_{h \sigma_{2}}^{*}$, such a $z^{*}$ cannot be an equilibrium allocation. Hence $z_{h \sigma_{1}}^{*}=z_{h \sigma_{2}}^{*}=z_{h r}^{*}$ for all $h=1, \ldots, H$. Note that this implies that in an equilibrium, household $h$ consumes the same allocation $z_{h r}^{*}$ across all individual states $s$, i.e. it achieves full insurance. Since $p^{*}$ supports the equilibrium allocation $z^{*}$, and $z_{h \sigma_{1}}^{*}=z_{h \sigma_{2}}^{*}$ it follows that $p_{\sigma_{1}}^{*}=p_{\sigma_{2}}$ when $r\left(\sigma_{1}\right)=r\left(\sigma_{2}\right)$, because utilities are assumed to be $C^{2}$ and, in particular, to have a unique gradient at each point which, by optimality, must be collinear both with $p_{\sigma_{1}}^{*}$ and with $p_{\sigma_{2}}^{*}$, i.e. $p_{\sigma_{1}}^{*}=p_{\sigma_{2}}^{*}=p_{r}^{*}$. This implies that at an equilibrium, household $\mathrm{h}$ of type $i$ faces the same prices $p_{\mathrm{r}}^{*}$ at any $\sigma$ with $r(\sigma)=r$. Since all household $\mathrm{h}$ of type $\mathrm{i}$ have the same strictly concave utilities (2), and they face the same prices $p_{r}^{*}$ at each $\sigma$ with $r(\sigma)=r$, they will choose the same fully insured consumption bundle $z_{\sigma}^{i *}=z_{r}^{i *}$ for all $\sigma$ with $r(\sigma)=r . \diamond^{2}$

We now consider the general case, which allows for $\Pi^{i} \neq \Pi^{j}$ if $i \neq j$. Proposition 1 no longer holds, and the households of the Arrow-Debreu economy $E$ may not achieve full insurance at an equilibrium. However, we show that if the economy is regular and if there are two individual states, there is always one equilibrium at which prices are the same at all collective states leading to the same statistical state.

Definition: An economy $E$ is regular if at all equilibrium prices in $E$ the Jacobian matrix of first partial derivatives of its excess demand function has full rank [9]. Regularity is a generic property [8][9].

\footnotetext{
${ }^{2}$ Note that in the proof of Proposition 1 we did not use the assumption that agents' probability distributions give measure one to the set of collective states leading a a particular statistical state.
} 
Proposition 2 An Arrow-Debreu equilibrium allocation of the economy $E\left(p^{*}, z^{*}\right)$ is not fully insured if $\Pi^{h} \neq \Pi^{k}$ for some households $h, k$ with $U^{h} \neq U^{k}$ in 2. In particular, household $h$ has a different equilibrium allocation across collective states $\sigma_{1}$ and $\sigma_{2}$ with $r\left(\sigma_{1}\right)=r\left(\sigma_{2}\right)$. When $E$ is a regular economy and there are two individual states, one of the equilibrium prices $p^{*}$ must satisfy $p_{\sigma_{1}}^{*}=p_{\sigma_{2}}^{*}$ for all $\sigma_{1}, \sigma_{2}$ with $r\left(\sigma_{1}\right)=r\left(\sigma_{2}\right)$.

Proof. Suppose in contradiction that household $h$ of type $i$ is in fact fully insured so that $z_{h \sigma_{1}}^{*}=z_{h \sigma_{2}}^{*}$ for all $\sigma^{1}$ and $\sigma_{2}$ with $r\left(\sigma_{1}\right)=r\left(\sigma_{2}\right)$. Household $h$ 's consumption levels are $y_{s_{1} r}^{i}$ and $y_{s_{2} r}^{i}$ where $s_{1}=s\left(h, \sigma_{1}\right)$ and $s_{2}=s\left(h, \sigma_{2}\right)$. By assumption we have $y_{s_{1} r}^{i}=y_{s_{2} r}^{i}$. Now from 2 household $h$ 's marginal rate of substitution between

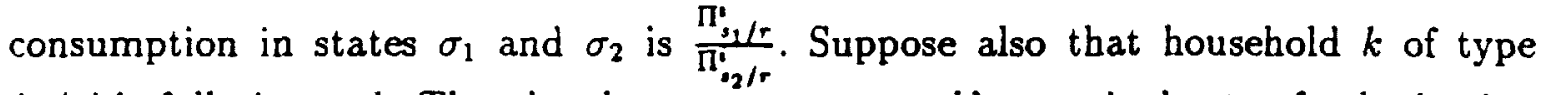
$j \neq i$ is fully insured. Then by the same argument $k$ 's marginal rate of substitution between consumption in states $\sigma_{1}$ and $\sigma_{2}$ is $\frac{\Pi_{3,1 r}^{\prime}}{\pi_{s_{2} / r}^{\prime}}$. But if different types have different probability distributions this is a contradiction as both face the same price vector.

Assume now that $E$ is regular, and that $S=2$. Consider two collective states $\sigma_{1}$ and $\sigma_{2}$ with $r\left(\sigma_{1}\right)=r\left(\sigma_{2}\right)$, and such that $\sigma_{1}$ differs from $\sigma_{2}$ only on the individual states of the two households $h_{1}$ and $h_{2}$ of the same type $i$ which are permuted, i.e., $s\left(h_{1}, \sigma_{1}\right)=s\left(h_{2}, \sigma_{2}\right)$ and $s\left(h_{2}, \sigma_{1}\right)=s\left(h_{1}, \sigma_{2}\right)$. Assume that there exists an equilibrium price for $E . p^{*} \in R^{N S^{H}}$, such that its components in states $\sigma_{1}$ and $\sigma_{2}$ are different, i.e. $p_{\sigma_{1}}^{*} \neq p_{\sigma_{2}}^{*}$. Define now a new price $p_{c}^{*} \in R^{N S^{H}}$, called a "conjugate" of $p^{*}$, which differs from $p^{*}$ only in its coordinates in states $\sigma_{1}$ and $\sigma_{2}$, which are permuted as follows: $\forall \sigma \neq \sigma_{1}, \sigma_{2}, p_{c \sigma}^{*}=p_{\sigma}^{*}, p_{c \sigma_{1}}^{*}=p_{\sigma_{2}}^{*}$, and $p_{c \sigma_{2}}^{*}=p_{\sigma_{1}}^{*}$. We shall now show that $p_{c}^{*}$ is also an equilibrium price for the economy $E$. At $p_{c}^{*}$, household $h_{1}$ has the same endowments and faces the same prices in states $\sigma_{1}$ and $\sigma_{2}$ as it did at states $\sigma_{2}$ and $\sigma_{1}$ respectively at price $p^{*}$; at all other states $\sigma \in \Omega, h_{1}$ faces the same prices and has the same endowments facing $p^{*}$ and facing $p_{c}^{*}$. The same is true of household $h_{2}$. Furthermore $h_{1}$ and $h_{2}$ have the same utilities and probabilities at $\sigma_{1}$ and $\sigma_{2}$ because $r\left(\sigma_{1}\right)=r\left(\sigma_{2}\right)$ and probabilities are anonymous, and because $h_{1}$ and $h_{2}$ are of the same type $i$. Therefore the excess demand vectors of $h_{1}$ in states $\sigma_{1}$ and $\sigma_{2}$ at prices $p^{*}$ equal the excess demand vectors of $h_{2}$ in $\sigma_{2}$ and $\sigma_{1}$ respectively, at prices $p_{c}^{*}$, and at all other states $\sigma \in \Omega$ the excess demand vectors of $h_{1}$ are the same at prices $p^{*}$ and $p_{c}^{*}$. Reciprocally: the excess demand vectors of $h_{2}$ in $\sigma_{1}$ and $\sigma_{2}$ at prices $p^{*}$ equal the excess demand vectors of $h_{1}$ in $\sigma_{2}$ and $\sigma_{1}$ respectively at prices $p_{c}^{*}$, and in all other states $\sigma$, the excess demand vectors of $h_{2}$ are the same as they are with prices $p^{*}$. Formally:

$$
\begin{aligned}
& z_{h_{1} \sigma_{1}}\left(p_{c}^{*}\right)=z_{h_{2} \sigma_{2}}\left(p^{*}\right), \quad z_{h_{1} \sigma_{2}}\left(p_{c}^{*}\right)=z_{h_{2} \sigma_{1}}\left(p^{*}\right) \\
& z_{h_{2} \sigma_{1}}\left(p_{c}^{*}\right)=z_{h_{1} \sigma_{2}}\left(p^{*}\right), \quad z_{h_{2} \sigma_{2}}\left(p_{c}^{*}\right)=z_{h_{1} \sigma_{1}}\left(p^{*}\right)
\end{aligned}
$$

and $\forall \sigma \in \Omega, \sigma \neq \sigma_{1}, \sigma_{2}$ :

$$
z_{h_{1} \sigma}\left(p^{*}\right)=z_{h_{1} \sigma}\left(p_{c}^{*}\right), z_{h_{2} \sigma}\left(p^{*}\right)=z_{h_{2} \sigma}\left(p_{c}^{*}\right)
$$


The excess demand vectors of all other households $h \neq h_{1}, h_{2}$ are the same for $p^{*}$ and $p_{c}^{*}$. Therefore at $p_{c}^{*}$ the aggregate excess demand vector of the economy is zero, so that $p_{c}^{*}$ is an equilibrium. The same argument shows that permuting the two components $p_{\sigma_{1}}^{*}, p_{\sigma_{2}}^{*}$ of a price $p^{*}$ at any two collective states $\sigma_{1}, \sigma_{2}$ leading to the same statistical state $r\left(\sigma_{1}\right)$ leads from an equilibrium price $p^{*}$ to another equilibrium price $p_{c}^{*}$. This is because if two collective states $\sigma_{1}$ and $\sigma_{2}$ lead to the same statistical state and there are two individual states $s_{1}$ and $s_{2}$ then there is a number $k>0$ such that $k$ households who are in $s_{1}$ in $\sigma_{1}$ are in $s_{2}$ in $\sigma_{2}$ and another $k$ households who were in $s_{1}$ in $\sigma_{2}$ are in $s_{2}$ in $\sigma_{1}$, while remaining in the same individual states otherwise. These two sets of $k$ households can be paired. For every pair of households, the above argument applies. Hence it applies to the sum of the demands, so that the new price $p_{c}^{*}$ is an equilibrium.

Now consider any regular economy $E$ with a finite number of equilibrium prices denoted $p_{i}^{*}, \ldots, p_{k}^{*}$. We shall show that there exists a $j \leq k$ s.t. $p_{j}^{*}$ assigns the same price vector to all collective states $\sigma_{1}, \sigma_{2}$ with $r\left(\sigma_{1}\right)=r\left(\sigma_{2}\right)$. Start with $p_{1}^{*}:$ if $p_{1}^{*}$ does not have this property, consider the first two collective states $\sigma_{1}, \sigma_{2}$ with $p_{i \sigma_{1}}^{*} \neq p_{i \sigma_{2}}^{*}$. Define $p_{c 1}^{*}$ as the conjugate of $p_{1}^{*}$ constructed by permuting the prices of the collective states $\sigma_{1}$ and $\sigma_{2}$. If $\forall j>1, p_{j}^{*}=p_{c l}^{*}$, then the number of price equilibria is even i.e. $k=2$; since however the number of price equilibria must be odd ${ }^{3}$, there must exist $p_{j_{1}}^{*}$ with $j_{1}>1$, and $p_{j_{1}}^{*} \neq p_{c 1}^{*}$. Consider the conjugate of $p_{j_{1}}^{*}$ with respect to the first two collective states $\sigma_{1}, \sigma_{2}$ which have different components in $p_{j_{1}}^{*}$, and denote this conjugate $p_{c j_{1}}^{*}$. Repeat the procedure until all equilibria are exhausted. In each step of this procedure, two different price equilibria are found. Since the number of equilibria must be odd, it follows that there must exist a $j \leq k$ for which all conjugates of $p_{j}^{*}$ equal $p_{j}^{*}$ : this is the required equilibrium which assigns the same equilibrium prices $p_{\sigma_{1}}^{*}=p_{\sigma_{2}}^{*}$ to all $\sigma_{1}, \sigma_{2}$ with $r\left(\sigma_{1}\right)=r\left(\sigma_{2}\right)$, completing the proof.$\diamond$

\section{Equilibrium \& incomplete markets for unknown risks}

Consider first the case where there are no assets to hedge against risk, so that the economy has incomplete asset markets. Individuals cannot transfer income to the unfavorable states. Examples are cases when individuals are not able to purchase health insurance. Market allocations are typically inefficient in this case, since individuals cannot transfer income from one state to another to equalize welfare across states. In an exhaustive formalization of all uncertainty in this economy states are described by all possible lists of all possible people with their states specified, i.e. by the elements $\sigma$ of the set $\Omega$. Which households will be in each state is unknown; each

${ }^{3}$ This follows from Dierker (1982) page 807 noting that his condition D is implied by our assumption that preferences are strictly increasing (see Dierker's remark following the statement of property $D$ on page 799 ). 
such list $\sigma$ can be seen as a different collective state with different probability. Each individual of type $i$ has a certain probability distribution over all possible collective states $\sigma, \Pi_{i}$. In each collective state $\sigma$ each individual is constrained in the value of her/his expenditures by her/his endowment (which depends on the individual state $s(h, \sigma)$ in that collective state). In this context, a general equilibrium of the economy with incomplete markets $E_{I}$ consists of a price vector $p^{*}$ with $N S^{H}$ components and $\mathrm{H}$ consumption plans $z_{h}^{*}$ with $V S^{H}$ components each, such that if individual $h$ is of type $i, z_{h}^{*}$ maximizes $W^{I}\left(z_{h}\right)$ as in 1 :

$$
W^{l}\left(z_{h}\right)=\sum_{\sigma \in \Omega} \Pi_{\sigma}^{i} U_{s(h, \sigma)}^{i}\left(z_{h \sigma}\right)
$$

subject to

$$
p_{\sigma}\left(z_{h \sigma}-e_{h \sigma}\right)=0 \text { for each } \sigma \in \Omega
$$

and

$$
\sum_{h=1}^{H}\left(z_{h}-e_{h}\right)=0
$$

The above economy $E_{l}$ is an extreme version of an economy with incomplete asset markets (see e.g. Geanakoplos [11]) because there are no markets to hedge against risks. There are $S^{H}$ budget constraints in $(\tau)$.

\section{Efficient allocations, mutual insurance \& secu- rities}

In this section we study the possibility of supporting Arrow-Debreu equilibria by combinations of Arrow securities and insurance contracts, rather than by using state contingent contracts. As already observed, this leads to a very significant economy in the number of markets needed. In an economy with no asset markets at all, such as $E_{I}$, the difficulty in supporting an Arrow-Debreu equilibrium arises because income cannot be transferred between states. On the basis of Propositions 1 and 2, we show that households can use Arrow securities defined on statistical states to transfer into each such state an amount of income equal to the expected difference between the value of Arrow-Debreu equilibrium consumption and the value of endowments. The expectation here is over individual states conditional on being in a given statistical state. The difference between the actual consumption-income gap given a particular individual state and its expected value is then covered by insurance contracts.

Theorem 1 Assume that all households in $E$ have the same probability $I$ over the distribution of risks in the population. Then any Arrow-Debreu equilibrium allocation $\left(p^{*}, z^{*}\right)$ of $E$ (and therefore any Pareto Optimum) can be achieved within the general equilibrium economy with incomplete markets $E_{I}$ by introducing into $E_{I}$ a total of 
I.A mutual insurance contracts to hedge against individual risk, and $A=\prod_{i}\left(\begin{array}{c}H_{i}+S+1 \\ S-1\end{array}\right)$ Arrow securities to hedge against collective risk. When $E$ is regular and $S=2$, this is also true even if households of different types have different probabilities over the distribution of risks in the population. In a regular economy with two individual states there is always an Arrow-Debreu equilibrium $\left(p^{*}, z^{*}\right)$ in $E$ which is achievable within the incomplete economy $E_{I}$ with the introduction of I.A mutual insurance contracts and A Arrow securities.

Proof. Consider first the case where all households have the same probabilities. By Proposition 1, an Arrow-Debreu equilibrium of $E$ has the same prices $p_{\sigma}^{*}=p_{\tau}^{*}$ and the same consumption vectors $z_{\sigma}^{i *}=z_{r}^{i *}$ for each $i$, at each collective state $\sigma$ with $r(\sigma)=r$. Define $\Omega(r)=\{\sigma \in \Omega: r(\sigma)=r\}$. The budget constraint 4 is

$$
p^{*}\left(z_{h}^{*}-e_{h}\right)=\sum_{\sigma} p_{\sigma}^{*}\left(z_{h, \sigma}^{*}-e_{h}\right)=\sum_{r} p_{r}^{*} \sum_{\sigma \in \Omega(r)}\left(z_{h \sigma}-e_{h \sigma}\right)=0
$$

Now individual endowments depend on individual states and not on social states, so that $e_{h \sigma}=e_{h s(\sigma)}=e_{h s}$ : furthermore by Proposition 1 equilibrium prices depend on $r$ and not on $\sigma$, so that for each $r$ the equilibrium consumption vector $z_{h \sigma}$ can be written as $z_{h s}$. The individual budget constraint is therefore $\sum_{r} p_{r}^{*} \sum_{s(r)}\left(z_{h},-e_{h}\right)$, where summation over $s(r)$ indicates summation over all individual states $s$ that occur in any collective state leading to $r$, i.e. that are in the set $\Omega(r)$. Let $\# \Omega(r)$ be the number of collective states in $\Omega(r)$. As $\Pi_{s / r}^{i}=r_{i s}$ is the proportion of households of type $i$ in state $s$ within the statistical state $r$, we can finally rewrite the budget constraint 4 of the household $h$ as:

$$
\# \Omega(r) \sum_{r} p_{r}^{*} \sum_{s} \Pi_{s / r}^{i}\left(z_{h s}-e_{h s}\right)=0
$$

From 2, the household's maximization problem is therefore: Maximize

$$
\sum_{s, r} \Pi_{s r} U^{h}\left(y_{s r}\right)
$$

subject to 9 , and the equilibrium allocation $z_{h}^{*}=\left(z_{r}^{i *}\right)$ solves this problem. Similarly, we may rewrite the market clearing condition 5 as follows:

$$
\sum_{h}\left(z_{h}^{*}-e_{h}\right)=\sum_{h}\left(z_{h \sigma}^{*}-e_{h s(\sigma)}\right)=0, \forall \sigma \in \Omega
$$

Rewriting 5 in terms of statistical states $r$, and within each $r$, individual states $s$ we obtain:

$$
\sum_{i} \sum_{s} r_{i s} H_{i}\left(z_{r}^{i *}-e_{s r}^{i}\right)=0, \forall r \in R
$$

or equivalently:

$$
\sum_{i, s} \Pi_{s / r}^{i} H_{i}\left(z_{r}^{i=}-e_{s r}^{i}\right)=0, \forall r \in R
$$


It follows that the allocation $z^{*}$ maximizes 2

Using these relations, we now show that any Arrow-Debreu equilibrium allocation $z^{*}=\left(z_{r}^{i *}\right)$ is within the budget constraints 7 of the economy $E_{I}$ for each $\sigma \in \Omega$, provided that for each $\sigma \in \Omega$ we add the income derived from an Arrow security $A_{r}, r=r(\sigma)$, and, given $r(\sigma)$, the income derived from mutual insurance contracts $m_{s r}^{i}=m_{s(\sigma) r(\sigma)}^{i}, s=1, \ldots, S$. We introduce $A$ Arrow securities and $I$. A mutual insurance contracts in the general equilibrium economy with incomplete markets $E_{I}$. The quantity of the Arrow security $A_{r}$ purchased by the $i$ th type of household in statistical state $r$, when equilibrium prices are $p^{*}$, is:

$$
a_{r}^{i *}=\sum_{s} \Pi_{s / r}^{i} p_{r}^{*}\left(z_{r}^{i *}-e_{s}^{i}\right)
$$

The quantity $a_{r}^{i *}$ has a very intuitive interpretation. It is the expected amount by which the value of equilibrium consumption exceeds the value of endowments, conditional on being in statistical state $r$. So on average, the Arrow securities purchased deliver enough to balance a household's budget in each statistical state. The balance is taken care of by the mutual insurance contracts. Note that 10 implies that the total amount of each Arrow security supplied is zero, i.e., $\sum_{i} H_{i} a_{r}^{i *}=0$ for all $r$, so that this corresponds to the initial endowments of the incomplete economy $E_{I}$. Furthermore, for each type $i, \sum_{\mathrm{r}} a_{\mathrm{r}}^{i *}=0$ by 9 , so that each household $h$ is within her/his budget in $E_{I}$.

We now introduce a mutual insurance contract as follows. The transfer made by an individual of type $i$ in statistical state $r$ and individual state $s$, when prices are $p_{r}^{*}$ :

$$
m_{s r}^{i *}=p_{r}^{*}\left(z_{r}^{i *}-e_{s}^{i}\right)-a_{r}^{i *}
$$

Note that, as remarked above, $m_{s r}^{i *}$ is just the difference between the actual incomeexpenditure gap, given that individual state $s$ is realized, and the expected incomeexpenditure gap $a_{r}^{i *}$ which is covered by Arrow securities. In each statistical state $r$, the sum over all $i$ and $s$ of all transfers $m_{s r}^{i *}$ equals zero i.e. the insurance premia match exactly the payments: for any given $r$,

$$
\sum_{i, \varrho} \Pi_{s / r}^{i} H_{i} m_{s r}^{i *}=\sum_{i, s} \Pi_{s / r}^{i} H_{i} p_{r}^{*}\left(z_{r}^{i *}-e_{s}^{i}\right)-\sum_{i} H_{i} a_{r}^{i *} \sum_{s} \Pi_{s / r}^{i}=0
$$

because $\sum, \Pi_{s / r}^{i}=1$. Therefore, the $\left\{m_{s r}^{i *}\right\}$ meet the definition of mutual insurance contracts. Finally, note that with $N$ spot markets, A Arrow securities $\left\{a_{r}\right\}$ and $I$ mutual insurance contracts $\left\{m_{s r}^{i}\right\}$

$$
\begin{gathered}
p_{r}^{*}\left(z_{r}^{i=}-e_{s}^{i}\right)=p_{r}^{*}\left(y_{r}^{i *}-e_{s}^{i}\right)=m_{s r}^{i *}+a_{r}^{i *}, \\
\forall \sigma \in \Omega \text { with } r(\sigma)=r, s=s(\sigma)
\end{gathered}
$$

so that 7 is satisfied for each $\sigma \in \Omega$. This establishes that when all households have the same probabilities over collective states, all Arrow-Debreu equilibrium allocation 
$z^{*}$ of $E$ can be achieved within the incomplete markets economy $E_{I}$ when $A$ Arrow securities and I.A mutual insurance contracts are introduced into $E_{l}$.

Consider now the case where the economy $E$ is regular, different types of households in $E$ have different probabilities over collective states, and $S=2$. By Proposition 2, we know that within the set of equilibrium prices there is one $p^{*}$ in which at all collective states $\sigma \in \Omega(r)$ for a given $r$, the equilibrium prices are the same, i.e. $p_{\sigma}^{*}=p_{r}^{*}$. In particular, if $E$ has a unique equilibrium $\left(p^{*}, z^{*}\right)$, it must have this property. It follows from the above arguments that the equilibrium $\left(p^{*}, z^{*}\right)$ must maximize 2 subject to 9 . Note however that now for the same $r, z_{s r}^{i *}$ may be different from $z_{s^{\prime} r}^{i}$ when $s \neq s^{\prime}$. Now define the quantity of the Arrow security $A_{r}$ purchased by a household of type $i$ in the statistical state $r$ by

$$
a_{r}^{i *}=\sum_{s} \Pi_{s / r}^{i} p_{r}^{*}\left(z_{s r}^{i *}-e_{s}^{i}\right)
$$

and the mutual insurance transfer made by a household of type $i$ in statistical state $r$ and individual state $s$, by

$$
m_{s r}^{i *}=p_{r}^{*}\left(z_{s r}^{i *}-e_{s}^{i}\right)-a_{r}^{i *}
$$

As before, $\sum^{r} a_{r}^{i *}=0$ and for any given $\mathrm{r}, \sum_{i, s} \Pi_{s / r}^{i} H_{i} m_{s r}^{i *}=0$, so that the Arrow securities purchased correspond to the initial endowments of the economy $E_{I}$ and at any statistical state the sum of the premia and the sum of the payments of the mutual insurance contracts match, completing the proof. $\diamond$

We can now formalize a statement made before about the efficiency of the institutional structure proposed in Theorem 1 by comparison with the standard ArrowDebreu structure of a complete set of state-contingent markets. We use here complexity theory, and in particular the concept of NP completeness. The key consideration in this approach to studying problem complexity is how fast the number of operations required to solve a problem increases with the size of the problem.

Definition: If the number of operations required to solve a problem must increase exponentially for any possible way of solving the problem, then the problem is called "intractable" or more formally, NP-complete. If this number increases polynomially, the problem is tractable. Further definitions are in Garey and Johnson [10].

The motivation for this distinction is of course that if the number of operations needed to solve the problem increases exponentially with some measure of the size of the problem, then there will be examples of the problem that no computer can or ever could solve. Hence there is no possibility of ever designing a general efficient algorithm for solving these problems. However, if the number of operations rises only polynomially then it is in principle possible to devise a general and efficient algorithm for the problem.

Theorem 2 investigates the complexity of the resource allocation problem in the Arrow-Debreu framework and compares this with the framework of Theorem 1 . We focus on how the problem changes as the economy grows in the sense that the number 
of households increases, and consider a very simple aspect of the allocation problem, which is as follows. Suppose that the excess demand of the economy $Z(p)$ is known. A particular price vector $p^{*}$ is proposed as a market clearing price. We wish to check whether or not it is a market clearing price. This involves computing each of the coordinates of $Z(p)$ and then comparing with zero. This involves a number of operations proportional to the number of components of $Z(p)$; we therefore take the rate at which the dimension of $Z(p)$ increases with the number of agents to be a measure of the complexity of the resource allocation problem. In summary: we ask how the difficulty of verifying market clearing increases as the number of households in the economy rises. We show that in the Arrow-Debreu framework this difficulty rises exponentially, whereas in the framework of Theorem 1 it rises only polynomially.

Theorem 2 Verifying market clearing is an intractable problem in an Arrow-Debreu economy, i.e., the number of operations required to check if a proposed price is market clearing increases exponentially with the number of households $H$. However, under the assumptions of Theorem 1. in the economy $E_{l}$ supplemented by I.A mutual insurance contracts and A Arrow securities, verifying market clearing is a tractable problem, i.e., the number of operations needed to check for market clearing increases only polynomially with the number of households.

Proof. The number of operations required to check that a price is market clearing is proportional to the number of market clearing conditions. In $\mathrm{E}$ we have $N S^{H}=$ $N S \sum H_{i}$ markets. Hence the number of operations needed to check if a proposed price is market clearing must rise exponentially with the number of households $H$. Consider now the case of $E_{l}$ supplemented by $I$.A mutual insurance contracts and A Arrow securities, where $A=\prod_{i}\left(\begin{array}{c}H_{i}+S+1 \\ S-1\end{array}\right)$. Under the assumptions of Theorem 1, by Propositions 1 and 2, we need only check for market clearing in one collective state associated with any statistical state, as if markets clear in one collective state leading to a certain statistical state they will clear in all collective states leading to the same statistical state. Hence we need to check a number of goods markets equal to $N . A$, plus markets for mutual insurance contracts and Arrow securities. Now

$$
A=\prod_{i}\left(\begin{array}{c}
H_{i}+S+1 \\
S-1
\end{array}\right)=\prod_{i}\left[\Phi_{i}\left(H_{i}, S\right)\right]
$$

where $\Phi_{i}\left(H_{i}, S\right)$ is a polynomial in $H_{i}$ of order $(S-1)$. Hence $A$ itself is a polynomial in $H_{i}$ whose highest order term depends on $\left(\prod_{i} H_{i}\right)^{S-1}$, completing the proof. $\diamond$

\section{Conclusions.}

We have defined an economy with unknown individual risks. In this context we have shown that ignorance of individual risks results in additional collective risks. Then we established that a combination of Arrow securities and mutual insurance 
contracts can be used to support Arrow-Debreu equilibria in economies with unknown risks. Furthermore we have shown that this institutional structure is efficient in the sense that it requires exponentially fewer markets that the standard approach via state-contingent commodities. In fact, the state-contingent problem is "intractable" with individual risks (formally, NP - complete) in the language of computational complexity, whereas our approach gives a formulation that is polynomially complex. This greatly increases the economy's ability to achieve efficient allocations. Another interesting feature of this institutional structure is the interplay of insurance and securities markets involved.

Individual risks whose probabilities are unknown, and indeed possibly unknowable, are an important category. They arise from new technologies (nuclear power or supersonic transport), from newly recognized environmental hazards (such as ozone depletion or climate change) and from newly recognized diseases (such as AIDS). In all of these examples, it is clear that there is little common ground on the risks to which individuals are exposed. In some of these examples the risks are quite unique and cannot be repeated to provide an opportunity for statistical inference about the true probabilities.

In examples such as those listed, our analysis implies that insurance companies issue insurance which depends on the statistical state. That is, they offer individuals an array of insurance contracts, one valid in each possible statistical state. Insurance contracts are therefore contingent on statistical states. Within each statistical state, of course, probabilities are known. Therefore companies are writing insurance only on known risks, something which is actuarially manageable. Individuals then buy the insurance that they want between statistical states via the markets for securities that are contingent on statistical states. Concretely, one would buy insurance against AIDS by (1) purchasing a set of AIDS insurance contracts each of which pays off only for a specified incidence of AIDS in the population as a whole, and (2) making bets via securities markets on the incidence of AIDS in the population. Likewise, one would obtain cover against an effect of climate change by (1) buying insurance policies specific to the health risks faced at particular levels of climate change, and (2) making bets on the level of climate change.

\section{References}

[1] Arrow, K.J. 1953. "The role of securities in an optimal allocation of risk-bearing". Econometrie, Proceedings of the Colloque sur les Fondements et Applications de la Theorie du Risque en Econometrie, Centre National de la Recherche Scientifique, Paris. English translation in Review of Economic Studies, 1964, 31, 91-96.

[2] Arrow, K.J. and R.C. Lind 1970. "Uncertainty and the evaluation of public investments". American Economic Review, 364-378. 
[3] Cass, D., G. Chichilnisky and H.M. Wu 1991. "Individual risks and mutual insurance." CARESS Working Paper \#91-27, Department of Economics, University of Pennsylvania.

[4] Chichilnisky, G. and H.M. Wu 1991. "Individual risk and endogenous uncertainty in incomplete asset markets". Working paper, Columbia University and Discussion Paper, Stanford Institute for Theoretical Economics.

[5] Chichilnisky G., J. Dutta and G.M. Heal 1991. "Options and price uncertainty." Working paper, Columbia Business School.

[6] Chichilnisky, G., G.M. Heal, P. Streufert and J. Swinkels 1992. "Believing in multiple equilibria". Working paper, Columbia Business School.

[7] Debreu, G. The Theory of Value. Wiley, New York.

[8] Debreu, G. 1970 "Economies with a finite set of equilibria". Econometrica, 38, $387-92$.

[9] Dierker, E. 1982. "Regular economies" in Handbook of Mathematical Economics Volume II, edited by K.J. Arrow and M.D. Intrilligator, North Holland.

[10] Gary, M.R. and D.S. Johnson 1979. Computers and Intractability: a Guide to NP-Completeness. W.H. Freeman and Company, New York.

[11] Geanakoplos, J. 1990. "An introduction to general equilibrium with incomplete asset markets". Journal of Mathematical Economics, 19, 1-38.

[12] Heal, G.M. 1991. "Risk management and global change". In B. Bohlin (ed) Proceedings of the First Vordic Conference on the Greenhouse Effect.

[13] Malinvaud, E. 1972. "The allocation of individual risk in large markets". Journal of Economic Theory, 4, 312-328.

[14] Malinvaud, E. 1973. "Markets for an exchange economy with individual risk". Econometrica, 3, 383-409 